\section{Dynamical Behaviors of an Euler Discretized Sliding Mode Control Systems}

\author{
Shaocheng Qu, Member, IEEE, Xiaohua Xia, Fellow, IEEE, and \\ Jiangfeng Zhang
}

\begin{abstract}
The dynamical behaviors of an Euler discretized sliding mode control (SMC) systems based on equivalent control strategy are studied. A periodic-2 orbit in steady state is found for the switching function of the Euler discretized SMC systems. The time steps for the switching function to converge toward the periodic-2 orbit are obtained. When the discretized SMC system is stable, it further shows that the system states of the SMC systems will also enter into some periodic-2 orbits, and these periodic-2 orbits are characterized by explicit analytic expressions. Finally, simulation examples are given to illustrate the theoretical results.
\end{abstract}

Keywords : Euler discretization, periodic orbits, sliding mode control.

\section{INTRODUCTION}

Sliding mode control (SMC) has been widely used in practical systems due to its simplicity and robustness in the presence of parameter variations and disturbances [1]. The research on discrete-time sliding mode control (DSMC) is important because most controllers are implemented in discrete-time [2]. There are two types of DSMC systems: one is the SMC of discrete-time systems [3]-[8], and the other is resulting from discretization of the SMC of continuous-time systems [9]-[17]. Our interest in this paper is the Euler discretization effect on the SMC of continuous-time systems.

For the discretization of the continuous-time SMC systems, the inherent limited switching frequency will cause system chattering (zigzagging) because of the freezing of the control signal during the sampling period [4]. Therefore, it is important to study the discretization effect on the SMC systems. Recently, the research on the zero-order-hold $(\mathrm{ZOH})$ discretization effect on the SMC systems has been actively carried out [9]-[13]. On the other hand, owing to their simple and practical characters, the Euler discretization methods are still commonly used for digital implementation of the SMC systems [6], [14]-[17], especially for servo-engineering systems despite its numerous drawbacks [6]. Therefore, it is important to study the dynamical behaviors of the Euler discretized SMC systems in order to evaluate the "deterioration" of performance. A periodic-2 orbit is found and bounds for the steady states are developed for an Euler discretized SMC system in [14]. The dynamical behaviors of the Euler

The work of S. Qu was supported by the NNSF of China (61074046/F030107), the Self-Determined International Cooperation Project of CCNU from the Colleges Basic Research and Operation of MOE (CCNU13F025), and the International Cooperation Project of China and the Ukraine (CU01-11). Recommended by Associate Editor M. L. Corradini.

S. Qu is with the Department of Electronics and Information Engineering, College of Physical Science and Technology, Central China Normal University, Wuhan 430079, China (e-mail: qushaocheng@ mail.ccnu.edu.cn).

X. Xia is with the Centre of New Energy Systems, Department of Electrical, Electronics and Computer Engineering, University of Pretoria, Pretoria 0002, South Africa (e-mail: xxia@ postino.up.ac.za).

J. Zhang was with the Centre of New Energy Systems, Department of Electrical, Electronics and Computer Engineering, University of Pretoria, Pretoria 0002, South Africa. He is now with the Department of Electronic and Electrical Engineering, University of Strathclyde, Glasgow G1 1XW, U.K. (e-mail: jiangfeng.zhang@strath.ac.uk). discretized system with respect to the sampling period are investigated in [15]. It should be mentioned that an implicit Euler numerical scheme is presented by redefining a multivalued sign function in [16] and [17], a chattering-free digital SMC with state observer and disturbance rejection is obtained, but it requires to solve a mixed linear complementarily problem (MLCP) at each step [17]. Although the implementation potential of the implicit Euler numerical scheme looks promising, it is yet to be widely accepted in practice.

In this note, a generalized SMC controller based on equivalent control method is employed to construct the Euler discretized SMC systems. Then the dynamics of the switching function along system trajectory are obtained. It is found that the switching function of the SMC systems converge to the periodic- 2 orbit in finite time steps, periodic- 2 orbit and the finite time steps are also calculated. Furthermore, periodic- 2 orbits of system states also are obtained when the discretized SMC systems are stable, and these periodic- 2 orbits are described by uniform analytic expressions. Finally, the analysis is illustrated by several simulations.

\section{DESIGN OF SMC SySTEMS BASED ON EQuivalent CONTROL METHOD}

Consider a general linear single input system in the controllable canonical form

$$
\dot{x}(t)=A x(t)+B u(t),
$$

where $x \in R^{n}$ is the state, $u \in R^{1}$ is the control, and

$$
\begin{aligned}
A & =\left[\begin{array}{cc}
0_{(n-1) \times 1} & I_{(n-1)} \\
-a_{1} & a_{p}
\end{array}\right], \quad B=\left[\begin{array}{c}
0_{(n-1) \times 1} \\
1
\end{array}\right], \\
a_{p} & =\left[-a_{2},-a_{3}, \ldots,-a_{n}\right] .
\end{aligned}
$$

Choose the switching function

$$
s(t)=c x(t),
$$

where the vector $c=\left[c_{1}, c_{2}, \ldots, c_{n-1}, 1\right]$ is to be designed such that the sliding mode reduced-order systems will achieve the desired dynamics when travelling along the switching manifold

$$
S=\{x(t) \mid c x(t)=0\} .
$$

Consider the sliding mode controller based on the equivalent control method [1]

$$
u(t)=-[c A x(t)+\beta s(t)+\alpha \operatorname{sgn}(s(t))],
$$

where $\alpha>0, \beta \geq 0$ are tuning parameters, $c x(t)$ is assumed to have relative degree " 1 " with respect to the system (1), and vector $c$ is chosen such that the following polynomial

$$
\lambda^{n-1}+c_{n-1} \lambda^{n-2}+\cdots+c_{2} \lambda+c_{1}=0
$$

is Hurwitz.

Note that controller (4) is the same form as given in [14]. The difference with [14] lies at the introduction of the term $\beta s(t)$, which allows to tune convergence rate of the switching function toward the switching manifold.

The following Lemmas are needed in the proof of Theorems 1-2.

Lemma 1 [12], [18]: For a one-dimensional delta-modulated feedback system in the form of discrete-time dynamics: $x^{+}=f(x) \triangleq$ $a x-\Delta \operatorname{sgn}(a x)$, where $x^{+}$denotes the system state at the next discrete-time step, $a$ and $\Delta>0$ are real number, and $\operatorname{sgn}(x)$ is defined as

$$
\operatorname{sgn}(x)= \begin{cases}1 & \text { if } x \geq 0 \\ -1 & \text { if } x<0\end{cases}
$$


The following results are valid:

(a) If $|a|=1$, then $\Omega=[-\Delta, \Delta]$ is a global attractor on $(-\infty, \infty)$. Thereafter $\Omega$ is called an attractor.

(b) If $|a|<1$, then the global attractor $\Omega$ is a set of two points: $\{-\Delta /(1+|a|), \Delta /(1+|a|)\}$.

(c) If $0 \leq a<1$, then the two points: $\{-\Delta /(1+|a|), \Delta /(1+$ $|a|)\}$ are 2-periodic points; if $-1<a<0$, then $\{-\Delta /(1+$ $|a|), \Delta /(1+|a|)\}$ are (1-periodic) fixed points.

(d) If $0<a<1$, the characteristic index $k$ is the smallest nonnegative integer $m$ such that $x^{(m)}$ and $x^{(m+1)}$ have opposite signs, where

$$
k=\left\lfloor\log _{a}\left(\frac{\Delta}{\Delta+(1-a)\left|x_{0}\right|}\right)\right\rfloor,
$$

and $x_{0}$ is an initial point, $\lfloor *\rfloor$ denotes the floor operation, i.e., the maximal integer bounded below the real number $*$.

Lemma 2 [13]: Consider a discrete-time system of an $n$-th order

$$
x^{+}=A x-b u,
$$

where $x \in R^{n}$ is the state, $x^{+}$denotes the system state at the next discrete-time step, $u \in R^{1}$ is the scalar control, $A$ and $b$ are constant matrices of appropriate dimensions, $A$ is a stable matrix, i.e., the eigenvalues of $A$ are all located within the unit circle. The following results are valid:

(a) For an asymptotically L-periodic input sequence, there is a periodic orbit of period $\mathrm{L}$ for the system (6).

(b) This periodic orbit is globally attracting.

\section{DynAmiCAl BehaViors OF SMC SySTEMS BASED ON EULER Discretization}

To study the discretization effects on the SMC systems, it is assumed that the control (4) is digitized through the Euler discretization at the sampling moments

$$
u^{(k)}=-\left[c A x^{(k)}+\beta s^{(k)}+\alpha \operatorname{sgn}\left(s^{(k)}\right)\right],
$$

for all $t \in[k T,(k+1) T]$, where $T>0$ is a sampling period, symbols $u^{(k)}, x^{(k)}$ and $s^{(k)}$ denote $u(k T), x(k T)$ and $s(k T)$ for simplification, respectively. Then the switching function (2) under Euler discretization is defined by

$$
s^{(k)}=c x^{(k)} .
$$

A discrete-time transformation of the system (1) under Euler discretization is obtained as

$$
x^{(k+1)}=(I+T A) x^{(k)}+B T u^{(k)} .
$$

By substituting (7) into (9), we have

$$
x^{(k+1)}=\left(I+T A_{c}\right) x^{(k)}-B \alpha T \operatorname{sgn}\left(s^{(k)}\right),
$$

where $A_{c}=A-B c A-B \beta c$, and

$$
A_{c}=\left[\begin{array}{cc}
0_{(n-1) \times 1} & I_{(n-1)} \\
-\beta c_{1} & a_{c p}
\end{array}\right]
$$

in which $a_{c p}=-c_{1}-\beta c_{2}, \ldots,-c_{n-2}-\beta c_{n-1},-c_{n-1}-\beta$.

In the following, we will investigate the Euler discretization effects of the switching function (8) and system states of the Euler discretized SMC systems (10).

\section{A. Discretization Behaviors of the Switching Function Along Trajectory}

Let us calculate the switching function of the SMC systems starting from any initial point.

Lemma 3: For system (1) with the switching function (2) under the sliding mode controller (4), assume that Euler discretization is implemented, then the dynamics of the switching function along system trajectory is described by

$$
s^{(k+1)}=(1-\beta T) s^{(k)}-\alpha T \operatorname{sgn}\left(s^{(k)}\right) .
$$

Proof: Substituting (7) and (9) into (8) yields

$$
\begin{aligned}
s^{(k+1)} & =c x^{(k+1)} \\
& =c(I+T A) x^{(k)}+c B T u^{(k)} \\
& =s^{(k)}+c T A x^{(k)}-T\left[c A x^{(k)}+\beta s^{(k)}+\alpha \operatorname{sgn}\left(s^{(k)}\right)\right] \\
& =(1-\beta T) s^{(k)}-\alpha T \operatorname{sgn}\left(s^{(k)}\right) .
\end{aligned}
$$

This completes the proof of Lemma 3.

Compared with the DSMC systems studied in [14], dynamics of the switching function (12) involves a tuning convergence rate of the switching dynamics, which is more general (formula (12) will changed into an uniform convergence rate in [14] when $\beta=0$ ). It should be indicated that (12) is in exactly the same form as the famous discrete reaching law employed by [4], [6], and [8]. So the following results obtained are also suitable for the DSMC system designed by discrete reach law in [4].

In the sequel, we assume further $0<1-\beta T \leq 1$, which is always possible by selecting the tuning parameter $\beta$ and reducing the sampling step $T$. The following results can be obtained.

Theorem 1: Assume $0<1-\beta T \leq 1$, the following results are valid for the dynamics of the switching function (12):

1) When $\beta>0$, the switching function starting from any initial point will first cross the switching manifold in $k^{*}+1$ number of steps, where

$$
k^{*}=\left\lfloor\log _{(1-\beta T)}\left(\frac{\alpha}{\alpha+\beta\left|s^{(0)}\right|}\right)\right\rfloor
$$

and then eventually converge to a symmetric periodic- 2 orbit in steady state

$$
\left\{\frac{-\alpha T}{2-\beta T}, \frac{\alpha T}{2-\beta T}\right\}
$$

where $s^{(0)}$ is an initial point of the switching function.

2) When $\beta=0$, the switching function starting from any initial point will converge to an asymmetric periodic-2 orbit [14]

$$
\left\{s^{*}, s^{*}-\alpha T \operatorname{sgn}\left(s^{*}\right)\right\} \in[-\alpha T, \alpha T]
$$

in a finite number of steps

$$
k^{* *}=\left\lfloor\frac{\left|s^{(0)}\right|}{\alpha T}\right\rfloor
$$

where $s^{*}$ is the first point that the switching function enters into the interval $[-\alpha T, \alpha T]$.

Proof: (a) When $\beta>0$, then $0<1-\beta T<1$ in (12). By Lemma $1(\mathrm{a}-\mathrm{c})$, the switching function starting from any initial point will converge to a symmetric periodic-2 orbit (14). By Lemma 1(d), the sequence $\left\{s^{(k)}\right\}$ begins to change sign at the $k^{*}$-th steps, i.e., the switching function $s^{(k)}$ starting from any initial point will first cross the switching manifold in $k^{*}+1$ number of steps.

(b) When $\beta=0$, then $1-\beta T=1$ in (12). So (12) is simplified to

$$
s^{(k+1)}=s^{(k)}-\alpha T \operatorname{sgn}\left(s^{(k)}\right) \text {. }
$$


By Lemma 1(a), the interval $[-\alpha T, \alpha T]$ is a global attractor on $(-\infty, \infty)$ for (17). Furthermore, it follows from (17) that $s^{(k)}>$ $s^{(k+1)} \geq-\alpha T$ for all $s^{(k)} \geq 0$, and $\alpha T \geq s^{(k+1)}>s^{(k)}$ for all $s^{(k)}<0$. It shows that the switching function starting from any initial point will be monotonically attracted to the interval $[-\alpha T, \alpha T]$.

Assume $s^{*}$ is the first point that the switching function starting from the initial point $s^{(0)}$ enters into the interval $[-\alpha T, \alpha T]$ at the $k$-th step, i.e., $s^{(k)}=s^{*} \in[-\alpha T, \alpha T]$, then the next step is $s^{(k+1)}=$ $s^{*}-\alpha T \operatorname{sgn}\left(s^{*}\right) \in[-\alpha T, \alpha T]$.

In the following, we investigate $s^{(k+2)}$ from two cases. If $0 \leq s^{*} \leq$ $\alpha T$, then

$$
\begin{aligned}
s^{(k+2)} & =s^{*}-\alpha T \operatorname{sgn}\left(s^{*}\right)-\alpha T \operatorname{sgn}\left(s^{*}-\alpha T \operatorname{sgn}\left(s^{*}\right)\right) \\
& =s^{*}-\alpha T+\alpha T \\
& =s^{*}
\end{aligned}
$$

Similarly, if $-\alpha T \leq s^{*}<0$, then $s^{(k+2)}=s^{*}$. From the above two cases, it is clear that the switching function of the closed-loop systems will directly enter into the periodic-2 orbit defined in (15) once the switching function enters into the interval $[-\alpha T, \alpha T]$.

In the sequel, (16) will be proved for the following two possible cases: $s^{(0)} \geq 0$ and $s^{(0)}<0$.

Assume $s^{(0)} \geq 0$, and $s^{(k)}$ are non-negative for all $k \leq m$ before the switching function of the closed-loop systems first cross the switching manifold. It follows from (17) that

$$
\begin{aligned}
s^{(1)}= & s^{(0)}-\alpha T, \\
s^{(2)}= & s^{(1)}-\alpha T=s^{(0)}-2 \alpha T, \\
& \vdots \\
s^{(m)}= & s^{(m-1)}-\alpha T=s^{(0)}-m \alpha T \\
= & \left|s^{(0)}\right|-m \alpha T .
\end{aligned}
$$

Similarly, assume $s^{(0)}<0$, then $s^{(k)}$ are negative for all $k \leq m$, then $s^{(m)}=-\left(\left|s^{(0)}\right|-m \alpha T\right)$.

In the above two cases, it is straightforward to verify that the real number $m^{*}=\left|s^{(0)}\right| / \alpha T$ satisfies $\left|s^{(0)}\right|-m^{*} \alpha T=0$. It shows that the switching function will start to enter into the interval $[-\alpha T, \alpha T]$ in $k^{* *}=\left\lfloor m^{*}\right\rfloor$ integer number of steps. Once the switching function enters into the interval $[-\alpha T, \alpha T]$, the switching function will enter into the periodic- 2 orbit (15) in $k^{* *}$ number of steps. This completes the proof of Theorem 1 .

Remark 1: If $\beta>0$ in (12), the switching function of the SMC systems starting from any initial point will begin to cross the switching manifold in the finite number of steps given in (13), and then eventually enter into the symmetric periodic-2 orbit (14) in steady state. If $\beta=0$ in (12), the switching function of the SMC systems starting from any initial point will converge to the asymmetric periodic- 2 orbit (15) in the number of steps defined in (16).

\section{B. Periodic-2 Orbits for SMC System States}

Now consider dynamic behaviors of the discretized SMC systems (10). From Lemma 2, the following results can be obtained.

Theorem 2: If $\beta>0$ and $\rho\left(I+T A_{c}\right)<1$, i.e., all eigenvalues of the matrix $\left(I+T A_{c}\right)$ defined in (10) are located within the unit circle, then the system states of the SMC systems (10) will converge to the symmetric periodic-2 orbits

$$
\left\{-\left(2 I+T A_{c}\right)^{-1} B \alpha T,\left(2 I+T A_{c}\right)^{-1} B \alpha T\right\} .
$$

Proof: When $\rho\left(I+T A_{c}\right)<1$, the matrix $I+T A_{c}$ of the discrete systems (10) is a stable matrix. According to Theorem 1, the input of the systems (10), i.e., the sequence $\left\{\operatorname{sgn}\left(s^{(k)}\right)\right\}$, is a periodic-2 orbit $\{-1,1\}$ in steady state. By Lemma 2 , the output of systems (10) will also form periodic- 2 orbits in steady state. These periodic- 2 orbits are calculated below.

Assume that the switching function of the SMC systems has already reached a periodic- 2 orbit after the $k$-th step, i.e.,

$$
\operatorname{sgn}\left(s^{(k)}\right)=1, \quad \operatorname{sgn}\left(s^{(k+1)}\right)=-1, \quad \operatorname{sgn}\left(s^{(k+2)}\right)=1 .
$$

It follows from (10) that

$$
\begin{aligned}
x^{(k+1)} & =\left(I+T A_{c}\right) x^{(k)}-B \alpha T \operatorname{sgn}\left(s^{(k)}\right) \\
& =\left(I+T A_{c}\right) x^{(k)}-B \alpha T .
\end{aligned}
$$

and

$$
\begin{aligned}
x^{(k+2)} & =\left(I+T A_{c}\right) x^{(k+1)}-B \alpha T \operatorname{sgn}\left(s^{(k+1)}\right) \\
& =\left(I+T A_{c}\right) x^{(k+1)}+B \alpha T \\
& =\left(I+T A_{c}\right)^{2} x^{(k)}-\left(I+T A_{c}\right) B \alpha T+B \alpha T .
\end{aligned}
$$

Since the sequence $\left\{x^{(k)}\right\}$ are periodic-2 orbits in steady state, it follows that

$$
\begin{aligned}
x^{(k+2)} & =\left(I+T A_{c}\right)^{2} x^{(k)}-\left(I+T A_{c}\right) B \alpha T+B \alpha T \\
& =x^{(k)} .
\end{aligned}
$$

Note that matrix $2 I+T A_{c}$ is non-singular when $T$ is sufficiently small and the matrix $A_{c}$ in (11) is non-singular when $\beta>0$, solving (19) yields $x^{(k)}=\left(2 I+T A_{c}\right)^{-1} B \alpha T$, and

$$
\begin{aligned}
x^{(k+1)} & =\left(I+T A_{c}\right)\left(2 I+T A_{c}\right)^{-1} B \alpha T-B \alpha T \\
& =\left[\left(I+T A_{c}\right)\left(2 I+T A_{c}\right)^{-1}-I\right] B \alpha T \\
& =\left[\left(2 I+T A_{c}-I\right)\left(2 I+T A_{c}\right)^{-1}-I\right] B \alpha T \\
& =-\left(2 I+T A_{c}\right)^{-1} B \alpha T .
\end{aligned}
$$

It shows that the system states of the SMC system (10) will enter into the symmetric periodic- 2 orbits described in (18). This completes the proof of Theorem 2.

Remark 2: If the sampling time period $T$ is sufficiently small, the inequality $\rho\left(I+T A_{c}\right)<1$ is valid for $\beta>0$ [14].

Remark 3: Note that the matrix $I+T A_{c}$ in (10) can be expressed as shown in (20), at the bottom of the page. If $\beta=0$, then the first column of the matrix (20) is composed of zeros except for element " 1 " at the diagonal. It shows that there exists at least one eigenvalue "1", which contradicts with the condition $\rho\left(I+T A_{c}\right)<1$ in Theorem 2 .

For comparison, we will further describe system states with uniform form when $\beta=0$. Assume $\beta=0$, then the system (10) can be expressed as

$$
\begin{aligned}
x_{1}^{(k+1)} & =x_{1}^{(k)}+\Gamma y^{(k)}, \\
y^{(k+1)} & =\Phi y^{(k)}+B_{1} \alpha T \operatorname{sgn}\left(s^{(k)}\right) .
\end{aligned}
$$

$$
I+T A_{c}=\left[\begin{array}{ccccc}
1 & T & 0 & \ldots & 0 \\
0 & 1 & T & \cdots & 0 \\
\vdots & \vdots & \ddots & \ddots & \vdots \\
0 & 0 & \ldots & 1 & T \\
-T \beta c_{1} & -T\left(c_{1}+\beta c_{2}\right) & \ldots & -T\left(c_{n-2}+\beta c_{n-1}\right) & -T\left(c_{n-1}+\beta\right)
\end{array}\right]
$$


where $y \triangleq\left(x_{2}, x_{3}, \ldots, x_{n}\right)^{T}, \Gamma=[T 0 \cdots 0] \in R^{1 \times(n-1)}, B_{1}=\left[\begin{array}{ll}0 & 0\end{array}\right.$ $\cdots 1]^{T} \in R^{(n-1) \times 1}$, and

$$
\Phi=\left[\begin{array}{ccccc}
1 & T & 0 & \ldots & 0 \\
0 & 1 & T & \ldots & 0 \\
\vdots & \vdots & \ddots & \ddots & \vdots \\
0 & 0 & \ldots & 1 & T \\
-T c_{1} & -T c_{2} & \ldots & -T c_{n-2} & 1-T c_{n-1}
\end{array}\right]
$$

in which $\Phi \in R^{(n-1) \times(n-1)}$.

Remark 4: If $\beta=0$ and $\rho(\Phi)<1$, then a similar proof as Theorem 2 shows the system states $y=\left(x_{2}, x_{3}, \ldots, x_{n}\right)^{T}$ of the system (21b) will converge to the symmetric periodic- 2 orbits in steady state

$$
\left\{-(I+\Phi)^{-1} B_{1} \alpha T,(I+\Phi)^{-1} B_{1} \alpha T\right\} .
$$

In the following, we consider the state $x_{1}$ of the system (21a). It follows from (21a) that

$$
\begin{aligned}
x_{1}^{(k+2)} & =x_{1}^{(k+1)}+\Gamma y^{(k+1)} \\
& =x_{1}^{(k+1)}+T x_{2}^{(k+1)} \\
& =x_{1}^{(k)}+T\left(x_{2}^{(k)}+x_{2}^{(k+1)}\right) .
\end{aligned}
$$

Once system (21b) enters into symmetric periodic- 2 orbits defined in (22), then $x_{2}^{(k)}+x_{2}^{(k+1)}=0$. It further shows that the state $x_{1}$ of the system (21a) will also enter into a periodic-2 orbit in steady state. From (8), one has

$$
s^{(k)}=c x^{(k)}=c_{1} x_{1}^{(k)}+c_{p} y^{(k)}
$$

where $c_{p}=\left[c_{2}, \cdots, c_{n-1}, 1\right]$. Note that $c_{1} \neq 0$, then

$$
x_{1}^{(k)}=c_{1}^{-1}\left(s^{(k)}-c_{p} y^{(k)}\right) .
$$

By Theorem 1(b), the switching function starting from any initial point will converge to the periodic- 2 orbit (15). In the meantime, system states $y=\left(x_{2}, x_{3}, \ldots, x_{n}\right)^{T}$ in (21b) will also enter into symmetric periodic-2 orbits (22) in steady state. Substituting (15) and (22) into (24), we can obtain the following conclusion.

Remark 5: If $\beta=0$ and $\rho(\Phi)<1$, the state $x_{1}$ of the system (21a) will converge to an asymmetric periodic- 2 orbit $\left\{P_{1}, P_{2}\right\}$ or $\left\{P_{3}, P_{4}\right\}$, where

$$
\begin{aligned}
& P_{1}=c_{1}^{-1}\left[s^{*}+c_{p}(I+\Phi)^{-1} B_{1} \alpha T\right], \\
& P_{2}=c_{1}^{-1}\left[s^{*}-\alpha T \operatorname{sgn}\left(s^{*}\right)-c_{p}(I+\Phi)^{-1} B_{1} \alpha T\right], \\
& P_{3}=c_{1}^{-1}\left[s^{*}-c_{p}(I+\Phi)^{-1} B_{1} \alpha T\right], \\
& P_{4}=c_{1}^{-1}\left[s^{*}-\alpha T \operatorname{sgn}\left(s^{*}\right)+c_{p}(I+\Phi)^{-1} B_{1} \alpha T\right]
\end{aligned}
$$

and it is bounded by

$$
\begin{aligned}
\left|x_{1}^{(k)}\right| & =\left|c_{1}^{-1}\left(s^{(k)}-c_{p} y^{(k)}\right)\right| \\
& \leq\left|c_{1}^{-1} s^{(k)}\right|+\left|c_{1}^{-1} c_{p} y^{(k)}\right| \\
& \leq c_{1}^{-1} \alpha T+\left|c_{1}^{-1} c_{p}(I+\Phi)^{-1} B_{1}\right| \alpha T .
\end{aligned}
$$

\section{NUMERICAL EXAMPLES}

Consider a linear single input system in the controllable canonical form of (1), where

$$
A=\left[\begin{array}{ccc}
0 & 1 & 0 \\
0 & 0 & 1 \\
-1 & -1 & -1
\end{array}\right], \quad B=\left[\begin{array}{l}
0 \\
0 \\
1
\end{array}\right]
$$

with an initial state $x(0)=[0,0.5,0.31]^{T}$. The switching parameter in (2) and the SMC parameters in (4) are chosen as $c=[1,1,1], \alpha=1$,

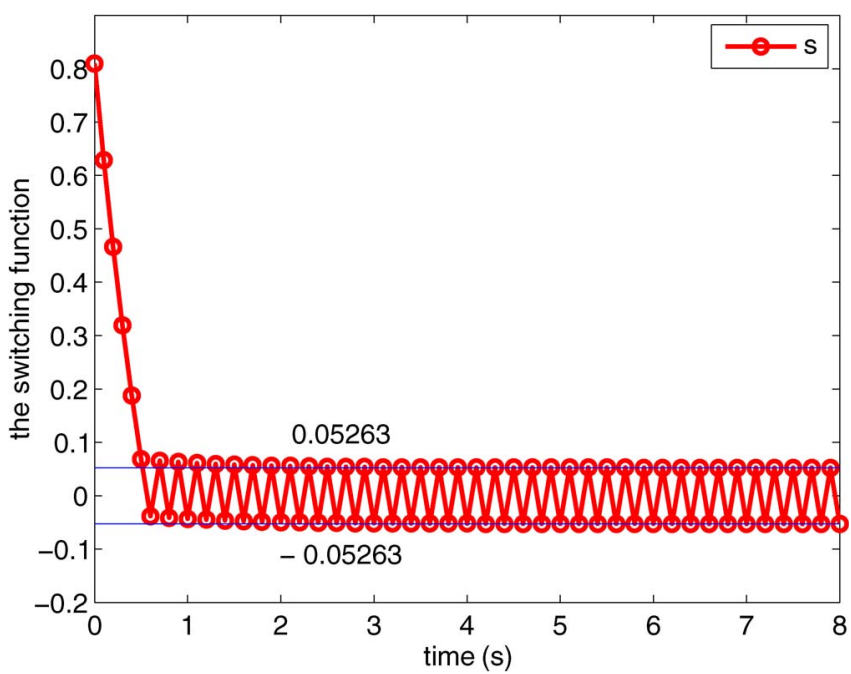

Fig. 1. Dynamics of the switching function with control parameter $\beta=1$.

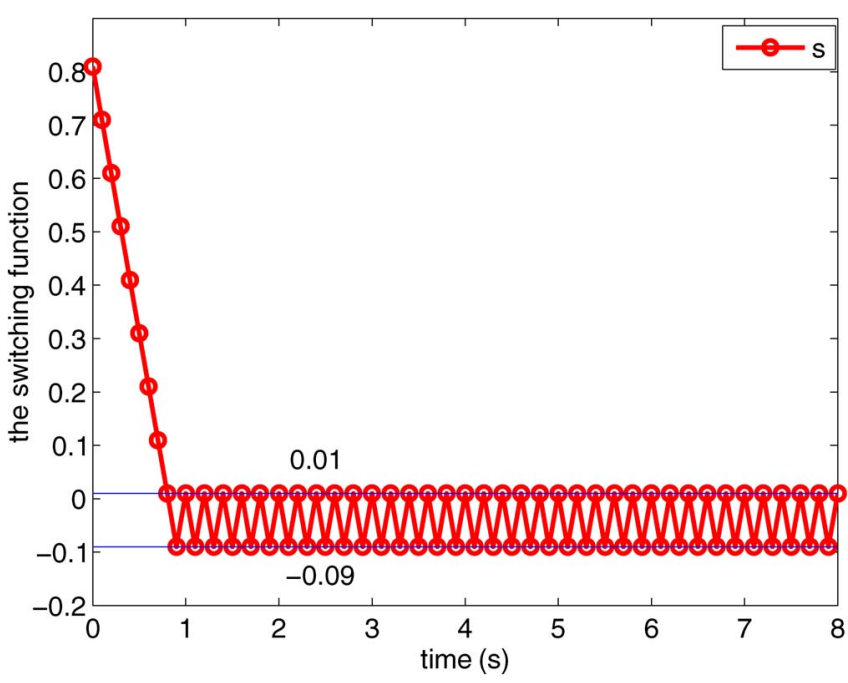

Fig. 2. Dynamics of the switching function with control parameter $\beta=0$.

respectively, and $\beta$ is a tuning control parameter. Sampling period is chosen as $T=0.1$, and the discrete SMC systems (10) is obtained by using the Euler discretization. In simulation, Matlab program is used in the following numerical examples.

\section{A. Dynamical Behaviors of the Switching Function Along Trajectory}

When the control parameter $\beta=1$ is chosen in (4), the dynamic behaviors of the switching function of the SMC systems are shown in Fig. 1. As stated in Theorem 1(a), the switching function of the SMC systems begins to cross the switching manifold in $k^{*}+1$ number of steps, where $k^{*}=5$ is the maximal integer bounded below the real number 5.6314 by (13) in Theorem 1. Furthermore, the switching function of the SMC systems eventually enters into a symmetric periodic- 2 orbit $\{-0.05263,0.05263\}$ described by (14).

When the control (4) with parameter $\beta=0$ is implemented, the dynamic behaviors of the switching function of the SMC systems are described in Fig. 2. As we observed from Fig. 2, the switching function of the SMC systems enters into an asymmetric periodic-2 orbit $\{-0.09,0.01\} \in[-0.1,0.1]$ in $k^{* *}$ number of steps by (15) and (16) in Theorem 1, where $k^{* *}=8$ is the maximal integer bounded below the real number 8.1 . 


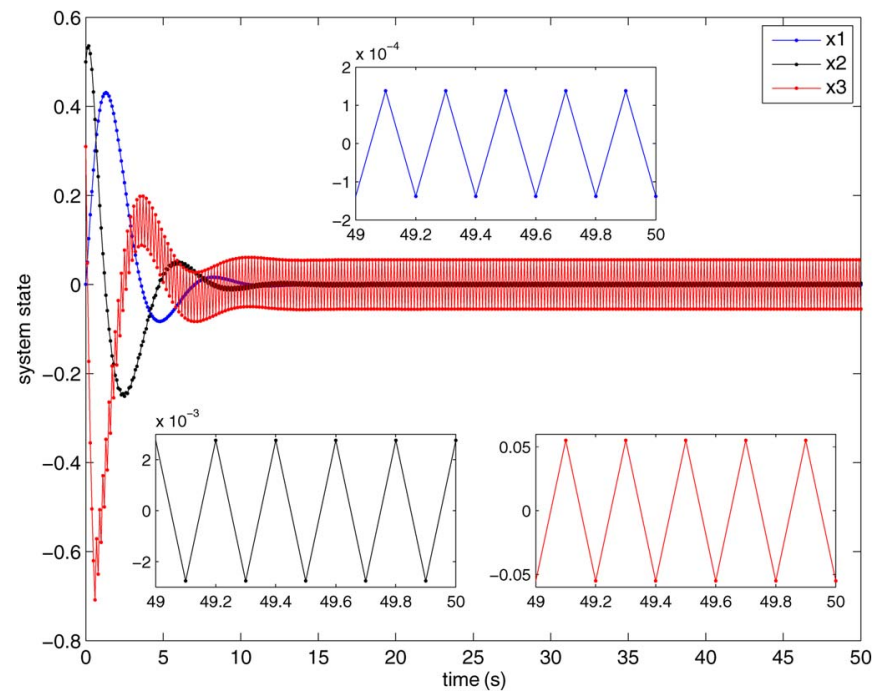

Fig. 3. System trajectories of the closed-loop SMC system with control parameter $\beta=1$.

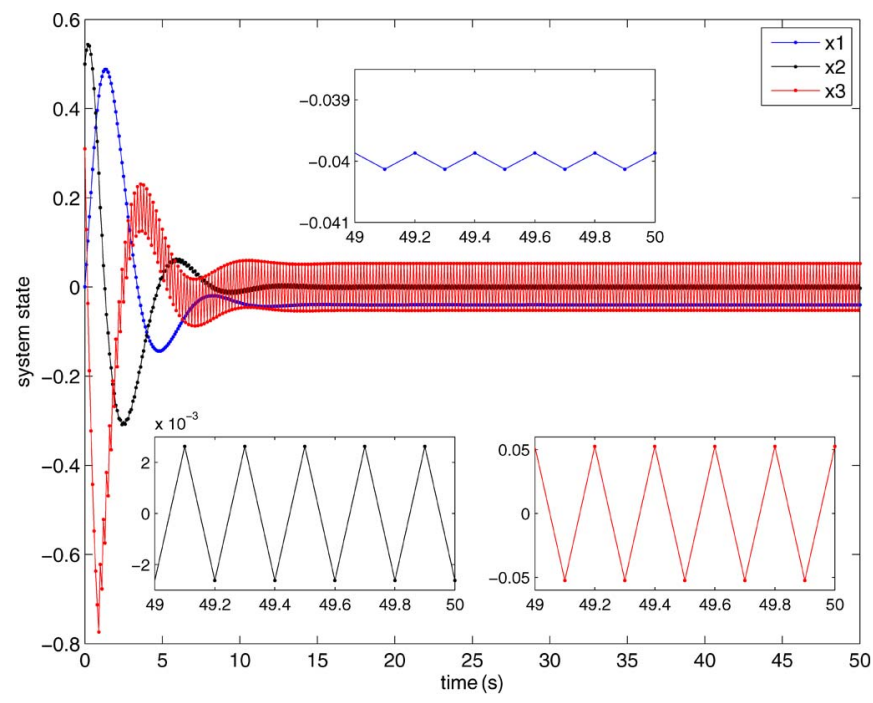

Fig. 4. System trajectories of the closed-loop SMC system with control parameter $\beta=0$.

\section{B. Periodic-2 Orbits for the SMC System States}

When control parameter $\beta=1$ is chosen in (4), the system states of the SMC systems are shown in Fig. 3. As stated in Theorem 2, the system states begin to converge toward the symmetric periodic- 2 orbits. After $42 \mathrm{~s}$ (420 steps), the system trajectories $x_{1}, x_{2}$ and $x_{3}$ enter into three symmetric periodic- 2 orbits in steady state $\{-0.00013814$, $0.00013814\},\{-0.00276281,0.00276281\}$, and $\{-0.05525625$, $0.05525625\}$, respectively.

When the control (4) with parameter $\beta=0$ is implemented, the system states of the SMC systems are described in Fig. 4. As stated in Remarks 4 and 5, the system trajectories begin to converge toward the periodic orbits. After $43 \mathrm{~s}$ (430 steps), the system trajectories $x_{2}$ and $x_{3}$ converge to the two symmetric periodic- 2 orbits $\{-0.0026247$, $0.0026247\}$ and $\{-0.052493,0.052493\}$, respectively. At the same time, the system trajectories $x_{1}$ also enter into an asymmetric periodic-2 orbit $\{-0.040131,-0.039869\}$. From Fig. 4 , it can be observed that periodic- 2 orbits of $x_{2}$ and $x_{3}$ in steady state are symmetric, but the periodic- 2 orbit of $x_{1}$ is asymmetric.

\section{CONCLUSION}

In this paper, the dynamical behaviors of the Euler discretization of the SMC systems based on equivalent control strategy are studied. The dynamical behaviors of the switching function of the Euler discretized SMC systems has been characterized. It is demonstrated that when the discretized SMC systems are stable, then the switching function and every state of the closed-loop systems will enter into respective periodic- 2 orbits. Furthermore, explicit analytic expressions are obtained to describe these periodic- 2 orbits. Future work will focus on the evaluation of performance "deterioration" of the Euler discretization of the SMC systems as well as the $\mathrm{ZOH}$ discretization of the SMC systems.

\section{ACKNOWLEDGMENT}

The authors would like to thank the anonymous reviewers and the associate editor for their many helpful comments.

\section{REFERENCES}

[1] V. I. Utkin, Sliding Modes in Control and Optimization. Berlin, Germany: Springer-Verlag, 1992.

[2] X. Yu, B. Wang, and X. Li, "Computer-controlled variable structure systems: The state-of-the-art," IEEE Trans. Ind. Informat., vol. 8, no. 2, pp. 197-205, Feb. 2012.

[3] O. Kaynak and A. Denker, "Discrete-time sliding mode control in the presence of system uncertainty," Int. J. Control, vol. 57, no. 5, pp. $1177-$ 1189,1993

[4] W. Gao, Y. Wang, and A. Homaifa, "Discrete-time variable structure control systems," IEEE Trans. Ind. Electron., vol. 42, no. 2, pp. 117-122, Apr. 1995.

[5] A. J. Bartoszewicz, "Discrete time quasi-sliding mode control strategies," IEEE Trans. Ind. Electron., vol. 45, no. 4, pp. 633-637, Apr. 1998.

[6] B. Veselić, B. Perunićić-Draženović, and Č. Milosavljević, "Improved discrete-time sliding-mode position control using Euler velocity estimation," IEEE Trans. Ind. Electron., vol. 57, no. 11, pp. 3840-3847, Nov. 2010.

[7] W. C. Su, S. V. Drakunov, and Ü. Özgüner, "An O (T2) boundary layer in sliding mode for sampled-data systems," IEEE Trans. Autom. Control, vol. 45, no. 3, pp. 482-485, Mar. 2000.

[8] M. C. Saaj, B. Bandyopashyay, and H. Unbehauen, "A new algorithm for discrete-time sliding-mode control using fast output sampling feedback," IEEE Trans. Ind. Electron., vol. 49, no. 3, pp. 518-523, Mar. 2002.

[9] B. Wang, X. Yu, and G. Chen, "ZOH discretization effect on single-input sliding mode control systems with matched uncertainties," Automatica, vol. 45, no. 1, pp. 118-125, Jan. 2009.

[10] B. Wang, X. Yu, and X. Li, "ZOH discretization effect on high-order sliding-mode control system," IEEE Trans. Ind. Electron., vol. 55, no. 11, pp. 4055-4064, Nov. 2008.

[11] X. Yu, B. Wang, Z. Galias, and G. Chen, "Discretization effect on equivalent control-based multi-input sliding-mode control systems," IEEE Trans. Autom. Control, vol. 53, no. 6, pp. 1563-1569, Jun. 2008.

[12] X. Xia and A. S. I. Zinober, "Delta-modulated feedback in discretization of sliding mode control," Automatica, vol. 42, no. 5, pp. 771-776, May 2006.

[13] X. Xia and A. S. I. Zinober, "Periodic orbits from delta-modulation of stable linear systems," IEEE Trans. Autom. Control, vol. 49, no. 8, pp. 13761380, Aug. 2004.

[14] Z. Galias and X. Yu, "Euler's discretization of single input sliding-mode control systems," IEEE Trans. Autom. Control, vol. 52, no. 9, pp. 17261730, Sep. 2007.

[15] Z. Galias and X. Yu, "Complex discretization behaviours of a simple sliding-mode control system," IEEE Trans. Circuits Syst. II, Exp. Briefs, vol. 53, no. 8, pp. 652-656, Sep. 2006.

[16] V. Acary and B. Brogliato, "Implicit Euler numerical scheme and chattering-free implementation of sliding mode systems," Syst. Control Lett., vol. 59, no. 5, pp. 284-293, May 2010.

[17] V. Acary, B. Brogliato, and Y. Orlov, "Chattering-free digital slidingmode control with state observer and disturbance rejection," IEEE Trans. Autom. Control, vol. 57, no. 5, pp. 1087-1101, May 2012.

[18] X. Xia and G. Chen, "On delta-modulated control: A simple system with complex dynamics," Chaos, Solitons and Fractals, vol. 33, no. 4, pp. 1314-1328, Aug. 2007. 